\title{
Pramipexole Dihydrochloride
}

National Cancer Institute

\section{Source}

National Cancer Institute. Pramipexole Dihydrochloride. NCI Thesaurus. Code C29374.

The hydrochloride salt of pramipexole, a benzothiazole derivative. As a nonergot dopamine agonist, pramipexole binds to D2 and D3 dopamine receptors in the striatum and substantia nigra of the brain. Compared to other dopamine agonists, the use of this agent may be associated with fewer dyskinetic side effects in treated subjects. ( $\mathrm{NCI04)}$ 